\title{
STRICTLY HYPOELLIPTIC SECOND ORDER DIFFERENTIAL OPERATORS
}

\author{
KAZUHIRO YAMAMOTO
}

\begin{abstract}
We shall show strict hypoellipticity of some second order differential operators which are generalized equations considered by Hörmander, Oleinik and Radkevix, using localized energy inequalities.
\end{abstract}

1. Result. Let $\Omega$ be an open set of $\mathbf{R}^{n}$ and $L(x, D)$ be a second order differential operator

$$
L(x, D)=X_{\alpha}(x, D) a^{\alpha \beta}(x) X_{\beta}(x, D)+X_{0}(x, D)+b(x),
$$

where $a^{\alpha \beta}(x), b(x) \in C^{\infty}(\Omega), a^{\alpha \beta}(x)=a^{\beta \alpha}(x)(\alpha, \beta=1, \ldots, N)$ and $X_{\alpha}(x, i D)$, $\left(\operatorname{Re} X_{0}\right)(x, i D)$ and $\left(\operatorname{Im} X_{0}\right)(x, i D)$ are real vector fields over $\Omega$. In (1.1) as well as the rest of this note we use the following convention of summation:

$$
\sum_{\alpha, \beta=1}^{N} A^{\alpha \beta} a_{\alpha} b_{\beta}=A^{\alpha \beta} a_{\alpha} b_{\beta} \text { and } \sum_{\alpha=1}^{N} A^{\alpha} a_{\alpha}=A^{\alpha} a_{\alpha}
$$

where $A^{\alpha \beta}, A^{\alpha}, a_{\alpha}$ and $b_{\beta}$ are functions or operators over $C^{\infty}(\Omega)$.

We shall define properly supported pseudo-differential operators $Y_{k}(x, D)(k=$ $0, \ldots, N+n)$ with the following symbols:

$$
\begin{aligned}
Y_{0}(x, \xi) & =\left(\operatorname{Im} X_{0}\right)(x, \xi), \quad Y_{\alpha}(x, \xi)=\left(\operatorname{Re} a^{\alpha \beta}\right)(x) X_{\beta}(x, \xi), \\
Y_{N+j}(x, \xi) & =\left(\partial\left(\operatorname{Re} a^{\alpha \beta}\right) / \partial x_{j}\right) X_{\alpha} X_{\beta}(x, \xi) \cdot|\xi|^{-1}
\end{aligned}
$$

where $\alpha=1, \ldots, N$ and $j=1, \ldots, n$. For a sequence $I=\left(i_{1}, \ldots, i_{s}\right)(0 \leq$ $\left.i_{k} \leq N+n\right)$ with the length $|I|=s$, we denote the iterated commutator $\left[Y_{i_{1}},\left[Y_{i_{2}}, \ldots,\left[Y_{i_{\text {s-1 }}}, Y_{i_{s}}\right] \ldots\right]\right]$ by $Y_{I}(x, D)$.

We impose the following conditions on $L$ :

(A.1) For any compact set $K$ of $\Omega$ there exists a proper and closed convex cone $\Gamma_{K} \subset\{z \in \mathbf{C} ; \operatorname{Re} z>0\} \cup\{0\}$ such that

$$
\left\{a^{\alpha \beta}(x) \eta_{\alpha} \eta_{\beta}:(x, \eta) \in K \times \mathbf{R}^{N}\right\} \subset \Gamma_{K} .
$$

(A.2) For any compact set $K$ of $\Omega$ there exists a constant $C_{k}$ such that

$$
\left|\left(\operatorname{Re} X_{0}\right)(x, \xi)\right|^{2} \leq C_{K}\left(\operatorname{Re} a^{\alpha \beta}\right)(x) X_{\alpha} X_{\beta}(x, \xi),
$$

where $(x, \xi) \in K \times \mathbf{R}^{N}$.

We shall use the following notation which is a microlocalized one of Oleinik and Radkevic [3].

DEFINITION. Let $\Gamma$ be an open conic subset of $T^{*}(\Omega) \backslash 0$. A second order differential operator $L(x, D)$ with a form (1.1) is of rank $n$ over $\Gamma$ if for any closed

Received by the editors June 12, 1981.

1980 Mathematics Subject Classification. Primary 35H05. 
subset of $\Gamma^{\prime}$ of $\Gamma$ such that $\Gamma^{\prime} \cap\left\{(x, \xi) \in T^{*}(\Omega) \backslash 0 ;|\xi|=1\right\}$ is a compact set, there exist a positive integer $\alpha=\alpha\left(\Gamma^{\prime}\right)$ and a positive constant $C=C\left(\Gamma^{\prime}\right)$ such that

$$
\sum_{|I| \leq \alpha}\left|Y_{I}(x, \xi)\right|^{2} \geq C|\xi|^{2}
$$

where $(x, \xi) \in \Gamma^{\prime}$. Here $Y_{I}(x, \xi)$ is the principal symbol of $Y_{I}(x, D)$.

THEOREM. Let $L(x, D)$ be a second order differential operator with a form (1.1), which satisfies assumptions (A.1) and (A.2). Then if $L(x, D)$ is of rank $n$ over $\Gamma$, for any $u \in D^{\prime}(\Omega)$ we have

$$
\mathrm{WF}(u) \cap \Gamma=\mathrm{WF}(L u) \cap \Gamma \text {. }
$$

As a consequence of the above theorem, we obtain the following:

COROLlaRY 1. Second order differential operators $L(x, D)$ considered in Hörmander [1] and Oleinik and Radkevic [3] are strictly hypoelliptic, if $L(x, D)$ is of rank $n$ over $T^{*}(\Omega) \backslash 0$.

Using our theorem, we can prove the following partial hypoellipticity:

CoROLlaRY 2. Let $\Omega=\Omega_{1} \times \Omega_{2}$, where $\Omega_{i}(i=1,2)$ is an open set of $\mathbf{R}^{n_{i}}$, and $\Xi$ be a conical neighbourhood of $\xi^{(2)}=0$ in $\mathbf{R}^{n_{i}}\left(n=n_{1}+n_{2}\right)$. If the second order differential operator $L(x, D)$ is of rank $n$ in $\Omega$, then $L$ is partially hypoelliptic on $x^{(1)}$, i.e., if $f \in C^{\infty}\left(\Omega_{1}, D^{\prime}\left(\Omega_{2}\right)\right), \mathrm{WF}(f) \subset \Omega \times B$ and $L u=f$, then $u \in C^{\infty}\left(\Omega_{1} ; D^{\prime}(\Omega)\right)$.

2. Preliminaries. First we shall state lemmas with respect to wave front set (see $\S 2.5$ of [2]).

LEMMA 2.1. A point $\left(x_{0}, \xi_{0}\right) \in T^{*}(\Omega) \backslash 0$ does not belong to $\mathrm{WF}(u)$ if and only if there exists a properly supported pseudo-differential operator $\chi(x, D)$ whose principal symbol does not vanish at $\left(x_{0}, \xi_{0}\right)$ such that $\chi(x, D) u \in C^{\infty}(\Omega)$.

LEMMA 2.2 (SEE PROPOSITION 2.5.9 OF [2]). If $u \in D^{\prime}(\Omega)$, we have $A(x, D) u \in$ $C^{\infty}(\Omega)$ for all properly supported pesudo-differential operators $A$ of $\Omega$ with

$$
\mathrm{WF}(A) \cap \mathrm{WF}(u)=0 .
$$

Next we shall cite some inequalities from [4]. Let $\tilde{Y}_{\alpha}(x, D)(\alpha=1, \ldots, N)$ and $\tilde{Y}_{N+j}(x, D)(j=1, \ldots, n)$ be properly supported pseudo-differential operators defined by the symbols $a^{\alpha \beta}(x) X_{\beta}(x ; \xi)$ and $\left(\partial a^{\alpha \beta}(x) / \partial x_{j}\right) X_{\alpha} X_{\beta}(x, \xi)|\xi|^{-1}$ respectively. Then just using the conditions (A.1) and (A.2), in [4] we proved the following:

LEMMA 2.3 (SEE LEMMA 2.3 OF [4]). (i) There exists a properly supported pseudo-differential operator $\Lambda_{0}$ of order 0 such that

$$
\sum_{k=1}^{N+n}\left(\left\|Y_{k} u\right\|^{2}+\left\|\tilde{Y}_{k} u\right\|^{2}\right) \leq C\left\{\left|\operatorname{Re}\left(\Lambda_{0} L u, u\right)\right|+\|u\|^{2}\right\},
$$

where $u \in C_{0}^{\infty}(K)$ and $\|\cdot\|$ and $($,$) are the norm and the inner product of L^{2}(\Omega)$, respectively.

(ii) For any properly supported pseudo-differential operator $\Lambda(x, D)$ of order $s$, there exist properly supported pseudo-differential operators $\Lambda_{k}(k=0, \ldots, N+k)$ 
of order s such that

$$
[L, \Lambda](x, D)=\sum_{k=1}^{n}\left(\Lambda_{k} \tilde{Y}_{k}\right)(x, D)+\Lambda_{0}(x, D) .
$$

Let $\chi$ and $\chi^{\prime}$ be properly supported pseudo-differential operators in $\Omega$ of order 0 such that the symbol of $\chi^{\prime}$ is identically 1 on some neighbourhood of the support of the symbol of $\chi$. Then we denote this relation between $\chi$ and $\chi^{\prime}$ by $\chi(x, D) \subset \subset$ $\chi^{\prime}(x, D)$. From now on we assume the supports of all properly supported pseudodifferential operators of order 0 denoted by $\chi, \chi^{\prime}$ or $\chi_{i}$ are contained in a subset $\Gamma^{\prime}$ of $\Gamma$, which satisfies the condition of the definition of $\S 1$.

The following lemma is easily proved by (2.1) and (2.2), and Lemma 2.3.

LEMMA 2.4. Let $\chi(x, D) \subset \subset \chi^{\prime}(X, D)$. Then we have

$$
\sum_{k=1}^{n+N}\left(\left\|Y_{k} \chi u\right\|_{s}^{2}+\left\|\tilde{Y}_{k} \chi u\right\|_{s}^{2}\right) \leq C\left(\left\|\chi^{\prime} L u\right\|_{s}^{2}+\left\|\chi^{\prime} u\right\|_{s}^{2}+\|u\|_{-r}^{2}\right) .
$$

Here $s \in \mathbf{R}, r$ is sufficiently large and $u \in C_{0}^{\infty}(K)$, where $K$ is a compact subset of $\Omega$.

LEMMA 2.5. Let $\chi(x, D) \subset \subset \chi^{\prime}(x, D)$ and $r$ be a sufficiently large number. Then for any $s \in \mathbf{R}$ and sufficiently large $r$ there exists a positive constant $\epsilon$,

$$
\|\chi u\|_{s+\epsilon} \leq C\left(\left\|\chi^{\prime} L u\right\|_{s}+\left\|\chi^{\prime} u\right\|_{s}+\|u\|_{-r}\right),
$$

where $u$ is $C_{0}^{\infty}(K)$.

PROOF. By (1.3) and a strong Gårding inequality we have

$$
\|\chi u\|_{\epsilon}^{2} \leq C\left(\sum_{|I| \leq \alpha}\left\|Y_{I} \chi^{\prime} u\right\|_{\epsilon-1}^{2}+\left\|\chi^{\prime} u\right\|^{2}+\|u\|_{-r}^{2}\right) .
$$

Using (2.3), (2.5) and the last inequality of p. 130 of [4], we can prove (2.4) as $s=0$. By the same way as the proof of Proposition 3.3 of [4], we can prove (2.4) for an arbitrary real number $S$.

3. Proof of theorem. Let $\varphi$ be an element of $C^{\infty}\left(\left\{x \in \mathbf{R}^{n} ;|x| \leq 1\right\}\right)$ with $\int \varphi(x) d x=1$. Then for $\delta<0$ we define the convolution operator

$$
\left(\varphi_{\delta} u\right)(x)=\delta^{-n} \int u(y) \varphi((y-x) / \delta) d y .
$$

Since $\varphi_{\delta}$ is a pseudo-differential operator with the symbol $\hat{\varphi}(\delta \xi)$, the set $\left\{\varphi_{\delta}(D)\right.$; $0 \leq \delta \leq 1\}$ is a bounded set of $L^{0}\left(\mathbf{R}^{n}\right)$. And we have $\operatorname{supp}\left(\varphi_{\delta} u\right) \subset \operatorname{supp} u+\delta$ and $u(x) \in \mathcal{E}^{\prime}(\Omega)$ belongs to $H_{s}(\Omega)$ if and only if $\left\|\varphi_{\delta} u\right\|_{s}<C$, where $C$ does not depend on $\delta$.

LEMMA 3.1. Let $u(x)$ be an element of $\mathcal{E}^{\prime}(\Omega)$ such that $\chi_{2} u(x)$ and $\left(\chi_{2} L u\right)(x)$ belong to $H_{s}(\Omega)$, then $\chi_{1} \tilde{Y}_{k} u(x)$ and $\chi_{1} Y_{k} u(x)$ belong to $H_{s}(\Omega)$, if $\chi_{1}(x, D) \subset \subset$ $\chi_{2}(x, D)$. 
ProOF. For simplicity we use the following terminology:

$$
A_{\delta}(u)=\sum_{k=1}^{N+n}\left(\left\|\varphi_{\delta} \chi_{1} Y_{k} u\right\|_{s}^{2}+\left\|\varphi_{\delta} \chi_{1} \tilde{Y}_{k} u\right\|_{s}^{2}\right)
$$

and

$$
B_{r}(u)=\left\|\chi_{2} L u\right\|_{s}^{2}+\left\|\chi_{2} u\right\|_{s}^{2}+\|u\|_{-r}^{2},
$$

where $r$ is a sufficiently large number. Let $\Lambda_{s}(x, D)$ be a properly supported elliptic pseudo-differential operator in $\Omega$ of order $s$. Then by an elliptic inequality we have

$$
A_{\delta}(u) \leq C \sum_{k=1}^{N+n}\left\{\left(\left\|\Lambda_{s} \varphi_{\delta} \chi_{1} \tilde{Y}_{k} u\right\|_{0}^{2}+\left\|\Lambda_{s} \varphi_{\delta} \chi_{1} Y_{k} u\right\|_{0}^{2}\right)+B_{r}(u)\right\}
$$

where in this proof constant numbers do not depend on $\delta$. Since $\left[Y_{k}, \Lambda_{s} \varphi_{\delta} \chi_{1}\right]\left(1-\chi_{2}\right)$ and $\left[\tilde{Y}_{k}, \Lambda_{s} \varphi_{\delta} \chi_{1}\right]\left(1-\chi_{2}\right)$ belong to $L^{-\infty}(\Omega)$, by (2.1) and (3.1), we have

$$
A_{\delta}(u) \leq C\left(\left|\left(\Lambda_{0} L\left(\Lambda_{s} \varphi_{\delta} \chi_{1} u\right), \Lambda_{s} \varphi_{\delta} \chi_{1} u\right)\right|+B_{r}(u)\right) .
$$

Using (2.2) and (3.2), there exist properly supported pseudo-differential operators $\Lambda_{s, j}(x, D)$ of order $s$ such that

$$
A_{\delta}(u) \leq C\left(\sum_{j=1}^{N+n}\left|\left(\Lambda_{s, j} \chi_{2} u, \tilde{Y}_{k}^{*} \Lambda_{s} \varphi_{\delta} \chi_{1} u\right)\right|+B_{r}(u)\right) .
$$

Since by the definitions of $Y_{k}$ and $\tilde{Y}_{k}^{*}=Y_{k}-i\left(\tilde{Y}_{k}-Y_{k}\right)+\tilde{Y}_{0}$, where $\tilde{Y}_{0}(x, D)$ is of order 0 , by (3.3) we have $A_{\delta}(u) \leq C B_{r}(u)$, using $x y \leq \epsilon x^{2}+y^{2} / \epsilon$ for any $\epsilon>0$ and nonnegative numbers $x$ and $y$. This completes the proof of Lemma 3.1.

LEMMA 3.2. Let $u(x)$ be an element of $\mathcal{E}^{\prime}(\Omega)$ such that $\chi_{2} u(x), \chi_{2} L u \in H_{s}(\Omega)$, then there exists a positive number $\epsilon$ such that $\chi_{1} u \in H_{s+\epsilon}(\Omega)$ if $\chi_{1}(x, D) \subset \subset$ $\chi_{2}(x, D)$.

PrOOF. We shall use the same terminology as that of Lemma 3.1. Since if $u \in \mathcal{E}^{\prime}(K)$, where $K$ is a compact set of $\Omega$, there exists a compact subset $K_{1}$ of $\Omega$ such that $\varphi_{\delta} u \in C_{0}^{\infty}\left(K_{1}\right)$, we can use (2.4). Thus

$$
\left\|\varphi_{\delta} \chi_{1} u\right\|_{s+\epsilon}^{2} \leq C\left\{\left\|\left[L, \varphi_{\delta} \chi^{\prime}\right] u\right\|_{s}^{2}+\left\|\left[\chi^{\prime}, L\right] \varphi_{\delta} u\right\|_{s}^{2}+B_{r}(u)\right\},
$$

where $C$ does not depend on $\delta$ and $\chi^{\prime}(x, D)$ is a properly supported pseudodifferential operator in $\Omega$ of order 0 such that $\chi_{1} \subset \subset \chi^{\prime} \subset \subset \chi_{2}$. Then since $\left[L, \varphi_{\delta} \chi_{2}\right] \in L^{-\infty}(\Omega)$ and $\chi^{\prime} Y_{k} u \in H_{s}(\Omega)(k=1, \ldots, N+n)$ by Lemma 3.1, we have $\left\|\varphi_{\delta} \chi_{1} u\right\|_{s+\epsilon}<C$, where $C$ does not depend on $\delta$. This completes the proof of Lemma 3.2.

Let us prove the theorem. Since it is well known WF $(L u) \subset \mathrm{WF}(u)$ for any pseudo-differential operator $L$ of $\Omega$ and $u \in D^{\prime}(\Omega)$, we shall prove $\mathrm{WF}(u) \subset$ WF $(L u)$, i.e., if $\left(x_{0}, \xi_{0}\right) \notin \mathrm{WF}(L u)$, then $\left(x_{0}, \xi_{0}\right) \notin \mathrm{WF}(u)$. Assume $\left(x_{0}, \xi_{0}\right) \in$ $T^{*}(\Omega) \backslash 0$ does not belong to WF $(L u)$. Then using a cut-off function which is not zero at $x_{0}$, we may assume $u \in \mathcal{E}^{\prime}(\Omega)$ and $\left(x_{0}, \xi_{0}\right) \notin \mathrm{WF}(L u)$. By Lemma 2.1 if we show a properly supported pseudo-differential operator $\chi(x, D)$ in $\Omega$ of order 0 such that $\chi u \in C^{\infty}(\Omega)$ and $\chi$ is elliptic near $\left(x_{0}, \xi_{0}\right)$, then $\left(x_{0}, \xi_{0}\right) \notin \mathrm{WF}(u)$. Here we assume $\left(x_{0}, \xi_{0}\right) \in \Gamma$. 
Since $\left(x_{0}, \xi_{0}\right) \notin \mathrm{WF}(L u)$, by Lemma 2.2, there exists an open conic neighbourhood $\tilde{\Gamma}$ of $\left(x_{0}, \xi_{0}\right)$ such that $\chi_{0}(x, D) L u \in C^{\infty}(\Omega)$ if the support of the symbol of $\chi_{0}$ contained in $\Gamma$. Let $\Gamma^{\prime}$ be an open conic subset of $\Gamma$ such that for some $\alpha>0, \Gamma_{1}=$ $\left\{(x, \xi) ; d((x, \xi), \Gamma)=\inf \left(\left|x-x^{\prime}\right|+|\xi /| \xi\left|-\xi^{\prime} /\right| \xi^{\prime}||<\alpha ;\left(x^{\prime}, \xi^{\prime}\right) \in \Gamma^{\prime}\right)\right\} \subset \tilde{\Gamma}$. Then we define $\Gamma_{n}=\left\{(x, \xi) ; d\left((x, \xi), \Gamma^{\prime}\right)<\alpha / n\right\}$ and $\chi_{n}(x, D)$ is a properly supported pseudo-differential operator such that the support of the symbol of $\chi h_{n}(n>2)$ is contained in $\Gamma_{2 n-1}$ and the symbol is equal to 1 on $\Gamma_{2 n}$. Since $\chi_{1} \supset \supset \chi_{2} \supset \supset \ldots$, inductively applying Lemma 3.2 to $\chi_{n-1}, \chi_{n}, u$ as $\chi_{1}=\chi_{n}$ and $\chi_{2}=\chi_{n-1}$, we have $\chi_{n} u \in H_{s+(n-1) \epsilon}(\Omega)$. If the support of the symbol of a properly supported pseudo-differential operator contained in $\Gamma^{\prime}$ then $\chi u \in H_{s+n \epsilon}(\Omega)$ for any positive integer $n$. This means $\chi u \in C^{\infty}(\Omega)$. Thus if $\chi(x, D)$ is elliptic in $\left(x_{0}, \xi_{0}\right)$, we have $\left(x_{0}, \xi_{0}\right) \notin \mathrm{WF}(u)$. This completes the proof of the theorem.

\section{REFERENCES}

1. L. Hörmander, Hypotelliptic second order differential equations, Acta Math. 119 (1967), 147-171.

2. _ Fourier integral operators. I, Acta Math. 127 (1971), 79-183.

3. O. A. Oleinik and E. V. Radkevic, Second order equations with non-negative characteristic form, Amer. Math. Soc., Providence, R.I., 1973.

4. K. Yamamoto, Hypoelliptic second order differential operators with complex coefficients, Studies in Anal., Adv. Math. Supplementary Studies (edited by G. C. Rota) 1 (1979), 123-133. 47907

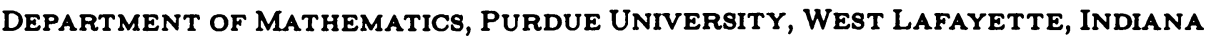
Japan

Current address: Department of Mathematics, Hokkaido University, 060 Sapporo N10,W8, 